(1)

CrossMark

\title{
Chronic cough: a disorder of response inhibition?
}

\author{
Stuart B. Mazzone (1) \\ Affiliation: Dept of Anatomy and Neuroscience, The University of Melbourne, Parkville, Australia.
}

Correspondence: Stuart B. Mazzone, Dept of Anatomy and Neuroscience, The University of Melbourne, Parkville, Victoria, 3010, Australia. E-mail: stuart.mazzonedunimelb.edu.au

@ERSpublications

The findings of Cho and co-workers suggest that chronic cough involves an imbalance between the level of peripheral drives that induce cough and the central mechanisms that are engaged to suppress cough http://ow.ly/tU1w30o9vBT

Cite this article as: Mazzone SB. Chronic cough: a disorder of response inhibition? Eur Respir J 2019; 53: 1900254 [https://doi.org/10.1183/13993003.00254-2019].

Many physicians will agree that chronic cough can be difficult to control and, for some patients, disease-modifying therapies fail to alleviate their cough. This is a condition now commonly referred to as refractory chronic cough and is present in a significant number of people in the community $[1,2]$. Our understanding of the mechanisms of cough has expanded dramatically in recent years [3], but it wasn't all that long ago when suggestions were rife that refractory chronic cough was a condition more likely "in the patient's head" than pathobiological in nature. Although the acceptance of refractory chronic cough as a real clinical entity is now well established, a study reported by $\mathrm{CHO}$ et al. [4], published in this issue of the European Respiratory Journal, suggests that we may need to revisit the notion that the problem resides in the brain, but not for the reasons once propagated.

It has been proposed that a unifying feature across most patients with chronic cough, including those who are not refractory to therapies, is a hypersensitivity to cough triggers (cough hypersensitivity syndrome) [5]. Thus, patients with chronic cough often cough more in response to low levels of known cough-evoking triggers (sometimes referred to as hypertussia) or in response to stimuli that do not usually cause cough in healthy individuals (allotussia) $[3,5]$. In the clinic, this can often be demonstrated with the use of cough sensitivity tests using inhaled stimuli including the chili extract capsaicin or adenosine triphosphate (ATP) $[6,7]$, in much the same way that airway hyperreactivity can be assessed in asthma patients through the use of methacholine challenges. In everyday life, cough hypersensitivity syndrome presents as troublesome cough triggered by seemingly innocuous events such as talking on the telephone, laughing, eating dry crumbly foods or smelling perfumes $[3,5]$. The long-held assumption is that this hypersensitivity must reflect a sensitisation of the peripheral terminals of airway nerves that induce cough when activated, probably secondary to an underlying inflammatory lung pathology.

The significance of the study reported by $\mathrm{CHO}$ et al. [4] is that it presents new evidence that peripheral sensitisation might not be the full story. In their study, patients with refractory chronic cough not only displayed evidence of cough hypersensitivity to inhaled capsaicin, but they also demonstrated little capacity to voluntarily suppress their cough during capsaicin challenges. This contrasts with the healthy participants in the study who could readily suppress capsaicin-evoked cough on command. Moreover, diminished cough suppression was related to the amount of daytime coughing objectively measured in patients using validated cough counting. Taken together, these data present a strong case suggesting that chronic cough involves both peripheral and central neural mechanisms. 
The observation that cough can be consciously influenced in humans is not new knowledge and most people will be able to recall a time that they suppressed a cough, perhaps during a concert, movie or in another social setting. Voluntary control of coughing has been studied behaviourally by several groups. Healthy participants can generally effectively suppress coughing when instructed to do so during cough challenge tests [8] or conversely regulate the intensity of their evoked cough efforts when asked to cough with weak or strong expiratory efforts [9]. This level of voluntary cough control points to the existence of brain networks that can be wilfully engaged to regulate cough motor patterning, presumably through pathways that descend from the "thinking" areas of the brain (the cerebral cortices) to the fundamental cough control centres in the brainstem and spinal cord (figure 1).

The central neural substrates that need to be engaged to voluntarily suppress coughing have been investigated in healthy humans using functional magnetic resonance imaging [10-12]. Such studies have revealed something interesting when it comes to cough suppression: the network that is engaged to suppress cough is strikingly similar to the network that is engaged to stop many kinds of motor actions. Within the brain exists a response inhibition neural circuit that allows for one motor programme to be stopped in order for another to be initiated. In behavioural tests this is often studied with the "go/no-go" task, involving participants finger tapping a button during repeated "go" commands and then having to quickly stop the task when "no-go" (or stop) signal is suddenly presented. Successful engagement of the response inhibition network in the brain, consisting of the right inferior frontal gyrus, right anterior insula and ventromedial prefrontal cortex, the dorsal mid-cingulate cortex and supplementary motor area, is needed to stop the finger tapping quickly when the no-go command appears [13]. Best characterised as a brake, response inhibition can be turned on outright or partially to suppress or pause a motor action, either in response to external cues or internal goals [14]. Slow response inhibition can be indicative of deficits in central inhibitory control. This same network becomes active in healthy people when told to hold their breath [15] or when challenged with inhaled capsaicin and told not to cough [10-12], suggesting that respiratory control and cough suppression requires normal response inhibition to be functional (figure 1). This was directly shown in a subsequent functional brain imaging study involving patients with refractory chronic cough in which an inability of patients to suppress cough was reflected in reduced levels of activity in component regions of their cough suppression network [16]. Whether chronic cough patients have generalised deficits in response inhibition is not known but could be easily assessed using go/no-go testing paradigms.
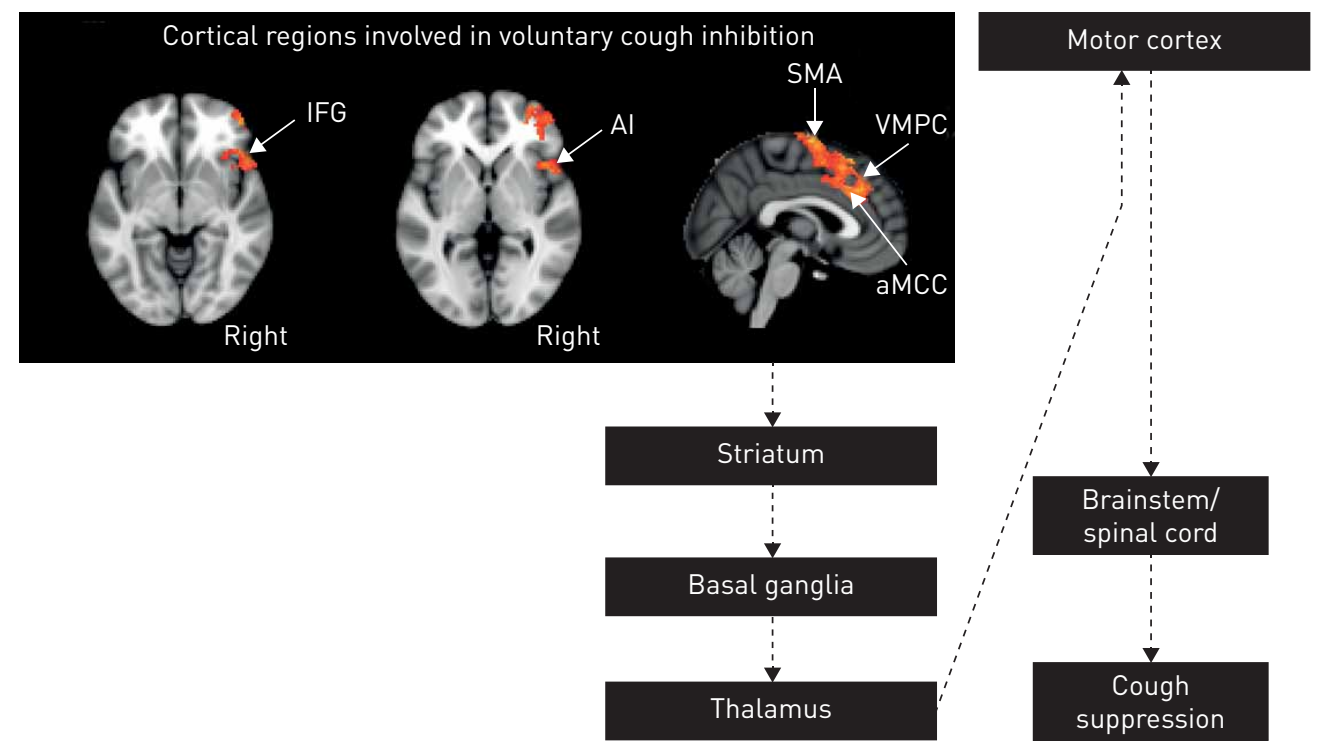

FIGURE 1 The putative voluntary cough suppression network. Functional brain imaging studies in healthy participants has revealed a cortical network involved in cough suppression. This network is remarkably similar to that involved in response inhibition, a brain process that is engaged to stop or pause motor responses. The response inhibition network incorporates deep brain nuclei, including the striatum, basal ganglia and thalamus, well known for their role in motor control. Thalamic regulation of the primary motor cortex allows motor movements, such as cough, to be suppressed. In patients with refractory chronic cough, the cortical components of this network are unable to be recruited [16] and this may explain why cough is unable to be effectively suppressed [4]. IFG: inferior frontal gyrus; Al: anterior insula; SMA: supplementary motor cortex; VMPC: ventromedial prefrontal cortex; aMCC: anterior-mid-cingulate cortex. Modified from [20] with permission. 
The findings of CHO et al. [4] are consistent with the notion that chronic cough can involve an imbalance between the level of peripheral drives that induce cough and the central mechanisms that are engaged to suppress cough. However, many questions remain. From a mechanistic point of view, it will be important to investigate whether loss of central suppression is involved in the development or maintenance (or both) of chronic cough. For example, it may be that the longer cough persists in a patient, the peripheral drivers that were originally responsible for cough induction become replaced with progressive diminution of central cough control. This could provide important insight into why cough can be so difficult to control in some patients. Alternatively, pre-existing deficits in the neural processes involved in response inhibition might be a predisposing characteristic of patients who go on to develop chronic cough. Furthermore, the response inhibition network is not the only brain process that can be engaged to modify coughing. Cognitive processes relating to the individual's expectations and beliefs are known to shape cough responses, most easily demonstrated with placebo-conditioning paradigms, but are also at play during situations that modify psychological states, including anxiety and fear [17-19]. The recruited brain networks needed for cognitive cough modulation are different to those involved in response inhibition $[20,21]$ and it is not clear whether their function is altered in patients with chronic cough. The fact that placebo responses are often reported in clinical trials of anti-tussives might suggest that these cognitive processes are preserved in cough patients, but carefully controlled investigations are ideally needed to assess this.

Finally, it will also be important to determine the relative contribution of peripheral versus central mechanisms underpinning chronic cough in any given patient, and whether patients with cough due to known causes similarly display lack of cough suppressive controls. Indeed, heterogeneous patient endotypes have significant impact on clinical management [22] and stratifying patients with chronic cough based on objective clinical presentation might afford better treatment success. New effective peripherally acting antitussive therapies are in clinical trial, including gefapixant (phase 3, ClinicalTrials.gov identifier NCT03449134), which specifically targets the P2X3 receptors for ATP on sensory nerves [23]. This is an exciting development. However, clinical data show that not all patients demonstrate benefits from this drug; in those that do, their cough is suppressed by $30-40 \%$ on average. The evidence is pointing to the notion that multiple mechanisms are responsible for chronic cough and these mechanisms are differentially expressed across individuals [22]. In this regard, it is interesting that CHO et al. [4] found that cough suppression tests proved a better discriminator of patients with refractory cough from healthy controls, compared to more traditional cough sensitivity testing. This observation might pave the way for the development of validated clinical challenge tests that allow for patients with predominately sensitive peripheral nerves to be distinguished from those who show lack of central control. In doing so, this should help guide disease management and therapy choice with respect to whether peripherally or centrally acting antitussive (and/ or behavioural) therapies are warranted. It is noteworthy, however, that voluntary cough suppression is not opioid-dependent [24], and as such new centrally acting antitussives that target and restore these central cough suppression networks will need to be developed. One exciting possibility is the centrally acting alpha-7 nicotinic receptor agonist bradanicline, which is currently in early phase 2 trials in refractory chronic cough patients (ClinicalTrials.gov identifier NCT03622216).

Although we have known for some time that the brain plays an important contributing role in other types of sensory hypersensitivities (such as chronic pain), the acceptance that this is also true for chronic cough, and probably for other symptoms of neural origin associated with pulmonary pathologies, has been slow. The work of $\mathrm{CHO}$ et al. [4] provides further impetus to pursue this important and under-investigated aspect of morbidity associated with pulmonary disease.

Conflict of interest: S.B. Mazzone has received fees for consultancy on gefapixant from Merck Sharpe and Dohme, outside the submitted work; and currently holds a Merck Investigator Initiated grant investigating ATP-evoked cough in chronic cough patients.

\section{References}

1 Song WJ, Chang YS, Faruqi S, et al. The global epidemiology of chronic cough in adults: a systematic review and meta-analysis. Eur Respir J 2015; 45: 1479-1481.

2 Gibson PG, Vertigan AE. Management of chronic refractory cough. BMJ 2015; 351: h5590.

3 Chung KF, McGarvey L, Mazzone SB. Chronic cough as a neuropathic disorder. Lancet Respir Med 2013; 1: 414-422.

4 Cho PSP, Fletcher HV, Turner RD, et al. Impaired cough suppression in chronic refractory cough. Eur Respir J 2019; 53: 1802203.

5 Morice AH, Millqvist E, Belvisi MG, et al. Expert opinion on the cough hypersensitivity syndrome in respiratory medicine. Eur Respir J 2014; 44: 1132-1148.

6 Ryan NM, Vertigan AE, Bone S, et al. Cough reflex sensitivity improves with speech language pathology management of refractory chronic cough. Cough 2010; 6: 5 .

7 Fowles HE, Rowland T, Wright C, et al. Tussive challenge with ATP and AMP: does it reveal cough hypersensitivity? Eur Respir J 2017; 49: 1601452. 

healthy volunteers. Respir Med 1993; 87: 379-382.

9 Hegland KW, Bolser DC, Davenport PW. Volitional control of reflex cough. J Appl Physiol 2012; 113: 39-46.

10 Farrell MJ, Cole LJ, Chiapoco D, et al. Neural correlates coding stimulus level and perception of capsaicin-evoked urge-to-cough in humans. NeuroImage 2012; 61: 1324-1335.

11 Farrell MJ, Koch S, Ando A, et al. Functionally connected brain regions in the network activated during capsaicin inhalation. Hum Brain Mapp 2014; 35: 5341-5355.

12 Mazzone SB, Cole LJ, Ando A, et al. Investigation of the neural control of cough and cough suppression in humans using functional brain imaging. J Neurosci 2011; 31: 2948-2958.

13 Jahfari S, Waldorp L, van den Wildenberg WP, et al. Effective connectivity reveals important roles for both the hyperdirect (fronto-subthalamic) and the indirect (fronto-striatal-pallidal) fronto-basal ganglia pathways during response inhibition. J Neurosci 2011; 31: 6891-6899.

14 Aron AR, Robbins TW, Poldrack RA. Inhibition and the right inferior frontal cortex: one decade on. Trends Cogn Sci (Regul Ed) 2014; 18: 177-185.

15 McKay LC, Adams L, Frackowiak RS, et al. A bilateral cortico-bulbar network associated with breath holding in humans, determined by functional magnetic resonance imaging. NeuroImage 2008; 40: 1824-1832.

16 Ando A, Smallwood D, McMahon M, et al. Neural correlates of cough hypersensitivity in humans: evidence for central sensitisation and dysfunctional inhibitory control. Thorax 2016; 71: 323-329.

17 Leech J, Mazzone SB, Farrell MJ. The effect of placebo conditioning on capsaicin-evoked urge to cough. Chest 2012; 142: 951-957.

18 Janssens T, Brepoels S, Dupont L, et al. The impact of harmfulness information on citric acid induced cough and urge-to-cough. Pulm Pharmacol Ther 2015; 31: 9-14.

19 Davenport PW, Vovk A, Duke RK, et al. The urge-to-cough and cough motor response modulation by the central effects of nicotine. Pulm Pharmacol Ther 2009; 22: 82-89.

20 Mazzone SB, McGovern AE, Farrell MJ. Endogenous central suppressive mechanisms regulating cough as potential targets for novel antitussive therapies. Curr Opin Pharmacol 2015; 22: 1-8.

21 Leech J, Mazzone SB, Farrell MJ. Brain activity associated with placebo suppression of the urge-to-cough in humans. Am J Respir Crit Care Med 2013; 188: 1069-1075.

22 Mazzone SB, Chung KF, McGarvey L. The heterogeneity of chronic cough: a case for endotypes of cough hypersensitivity. Lancet Respir Med 2018; 6: 636-646.

23 Abdulqawi R, Dockry R, Holt $\mathrm{K}$, et al. P2X3 receptor antagonist (AF-219) in refractory chronic cough: a randomised, double-blind, placebo-controlled phase 2 study. Lancet 2015; 385: 1198-1205.

24 Hutchings HA, Eccles R. The opioid agonist codeine and antagonist naltrexone do not affect voluntary suppression of capsaicin induced cough in healthy subjects. Eur Respir J 1994; 7: 715-719. 